5. to call on the museum-administrations to formulate and then to make publicly known their acquisition policy in relation to antiquities, with equal force for the acceptance of objects on loan or conservation: this policy includes the strong observance not to handle objects without a "pedigree" about their provenance;

6. to ask all museums and institutions interested in cultural exchanges to constantly inform the public about destruction of cultural heritage caused by illicit excavation or terrorism.
The resolution was accepted unanimously at Olympia the 4th of Sept. 2015 by Ashish Baheti, Sarika Baheti, Tung Ching, Angelos Delivorrias, Eleutherios Diamantaras, Hatto Fischer, Alexandros Hahalis, Dionysos Gangas, Wolf-Dieter Heilmeyer, Jane Ip, Way Jing, Li Junghong, J. Christian Kirsch, Leonidas Liu, Alexandros Mallias, George Manginis, Alexia Mercouris, Spyros Mercouris, Chico Sciuto, Robert Sidelsky, Panagiotis Skordas, Agne Vlavianos Arvanitis, Shelley Wache, Zang Xiaoming, Andreas Zaimis, Konstantinos A. Zaimis and many others more.

\title{
Rechtsprechung
}

\section{Zur Eigentumsvermutung für den Besitzer bei Beschlagnahme einer Sache}

Oberlandesgericht Köln, Urteil vom 6. August 2015 - 8 U 69/14

1. §1006 BGB vermutet die Gleichzeitigkeit von Besitz- und Eigentumserwerb, wenn nicht der Eigentumserwerb durch die Art des Besitzerwerbs ausgeschlossen ist.

2. Die vom Besitzerwerb ausgehende Eigentumsvermutung zugunsten eines früheren Besitzers wirkt ungeachtet des Wortlauts des $§ 1006$ Abs. 2 BGB über die Beendigung des Besitzes hinaus fort, bis sie widerlegt ist. (Leitsätze der Redaktion)

\section{Gründe:}

I.

- 1. Der Kläger begehrt die Herausgabe des auch als "Q" bezeichneten Bildes „P" des am 10. Juni 2010 verstorbenen Künstlers Prof. Q2. Die Beklagten sind dessen Erben. Der Erblasser hatte das Bild um 1969 entworfen und in den 1970er-Jahren gemalt. Anschließend hatte er es über drei Jahrzehnte in seinem Besitz und Eigentum. Am 4. September 2009 beschlagnahmte die Staatsanwaltschaft Köln dieses im Rahmen eines gegen den Kläger geführten Ermittlungsverfahrens in den Räumlichkeiten einer von diesem betriebenen Werbeagentur. Das Ermittlungsverfahren wegen des Vorwurfes des Diebstahls und der Hehlerei war durch einen Hinweis des Kunstspediteurs $L$ in Gang gesetzt worden. Mit Verfügung vom 21. Dezember 2009 gab die Staatsanwaltschaft das Bild an den Erblasser heraus. Am 14. Juli 2010 erhob sie gegen den Kläger Anklage wegen des Vorwurfes der Hehlerei; das Amtsgericht Köln lehnte durch Beschluss vom 1. März 2011 die Eröffnung des Hauptverfahrens aus tatsächlichen Gründen ab. Durch Beschluss vom 21. Dezember 2011 stellte das Landgericht Köln überdies fest, dass die Herausgabe des Bildes an den Erblasser rechtswidrig war.

Der Kläger hat behauptet, er habe im September des Jahres 2007 unmittelbar vom Erblasser Besitz und Eigentum an dem Bild erworben. Er habe den Erblasser in den Jahren 2006/2007 kennengelernt. Etwa Mitte 2007 sei anlässlich eines Atelierbesuches das Gespräch auf den Erwerb eines Werkes des Erblassers durch den Kläger gekommen. Nach wiederholter Nachfrage habe der Erblasser sich bereit erklärt, das streitgegenständliche Bild zu einem Preis von 100.000 Euro an den Kläger zu verkaufen. Der Erblasser habe allerdings gefordert, dass der Kläger den Kaufpreis in bar zahle, das Bild nie in eine Auktion gebe, es ausschließlich privat halte und nur notfalls veräußere. Den vereinbarten Kaufpreis habe er - der Kläger - sich unter anderem mittels Darlehen verschafft. Er habe zunächst 40.000 Euro und in einem weiteren Termin Ende September 2007 den Restbetrag in bar an den Erblasser übergeben. Er habe mit diesem die Abholung des Bildes durch eine Hilfsperson vereinbart. Der Zeuge $\mathrm{H}$ habe den Transport durchgeführt. Dieser habe sich zu dem Atelier des Erblassers begeben, den Erblasser dort angetroffen und ihm erklärt, das an den Kläger verkaufte Bild abzuholen. Der Erblasser habe den Zeugen auf ein bereits verpacktes Bild von ungefähr 4 qm Größe verwiesen und hierzu erklärt, es handele sich um das Bild für den Kläger. Der Erblasser habe dem Zeugen H geholfen, das Bild auf die Ladefläche des Transporters zu legen. Das Bild habe sich hiernach ununterbrochen bis etwa in das Frühjahr 2009 in der Wohnung des Klägers befunden; in diesem Zeitraum sei es ausschließlich einem kleinen Kreis von Freunden und sehr guten Bekannten vorgestellt worden. Aufgrund seiner finanziellen Lage habe der Kläger das Bild ab 
Frühjahr 2009 in den benachbarten Räumlichkeiten seiner Werbeagentur ausgestellt, wo es bis zu seiner späteren Beschlagnahme durch die Staatsanwaltschaft ausgestellt geblieben sei.

Der Kläger hat beantragt,

1. die Beklagten als Gesamtschuldner zu verurteilen, das im Eigentum des Klägers stehende Gemälde "P"; 1969 (auch bezeichnet als: „Q") des Künstlers Prof. Q2 an den Kläger herauszugeben;

2. die Beklagten als Gesamtschuldner zu verurteilen, an den Kläger 5.798,16 Euro außergerichtliche Rechtsanwaltskosten zu erstatten

Die Beklagten haben beantragt, die Klage abzuweisen.

Sie haben eine durch den Erblasser erfolgte Übereignung des Bildes an den Kläger bestritten. Dieser habe den Erblasser nicht gekannt. Außerdem sei das Bild mit "Q3" und damit nicht vom Erblasser signiert. Dieser habe seine Werke stets erst mit Verkauf oder Schenkung signiert. Zudem habe der Zeuge T das Bild in den Jahren 2008 oder 2009 und damit nach dem von dem Kläger behaupteten Erwerbszeitpunkt im Atelier des Erblassers gesehen. Gleiches gelte für die Beobachtungen des Zeugen L2. Ferner habe der Erblasser gegenüber dem Zeugen T das Bild als unverkäuflich bezeichnet, weil es sehr wichtig für ihn sei. Der Erblasser habe überdies gegenüber der Zeugin Q4 das Bild nach der Beschlagnahme durch die Staatsanwaltschaft als sein Eigentum wiedererkannt. Er sei entsetzt darüber gewesen, dass es aus seinem Privatlager entwendet worden sei. Er habe erklärt, es habe noch nie eine Ausstellung gesehen; er habe es nicht verkauft. Gleiches habe der Erblasser anlässlich seiner polizeilichen Vernehmung am 14. Oktober 2009 bekundet. Dabei habe er das Bild ebenfalls als unverkäuflich bezeichnet und selbst mit 400.000 Euro taxiert. Entgegen den Schilderungen des klägerischen Zeugen $\mathrm{H}$ habe das Bild auch in einem Zelt auf dem Hof gestanden. Zudem habe es sich bei der Beschlagnahme entgegen früheren Zeitpunkten auf einem unsachgemäß zusammengezimmerten Holzrahmen befunden. Es sei unter Missachtung der ursprünglichen Justierung unsachgemäß aufgerahmt worden. Teile der Darstellung der Bildervorderseite hätten sich auf den Seiten des Rahmenkonstrukts wiedergefunden. Unzutreffend seien schließlich auch die Behauptungen des Klägers zum angeblichen Hintergrund der Barverkaufsabrede sowie die Angaben des Zeugen $\mathrm{H}$ zum Transport. Entgegen der Behauptung des Klägers habe der Erblasser keinen Exklusivvertrag mit einer Galerie unterhalten. Auch habe der Erblasser entgegen der Behauptung des Zeugen $\mathrm{H}$ dem Kläger aufgrund seiner schmerzenden Schulter nicht beim Tragen des Bildes helfen können.

2. Das Landgericht hat der Klage bis auf die geltend gemachten außergerichtlichen Rechtsanwaltskosten stattgegeben. Ein Anspruch folge aus § 985 BGB. Die Beklagten seien unstreitig Besitzer des Bildes. Der Kläger sei dessen Eigentümer. Davon sei aufgrund der Vermutung des $\S 1006$ Abs. 1 BGB auszugehen. Diese Vermutung sei von den Beklagten nicht widerlegt worden. Sie könnten sich nicht darauf berufen, dass der Kläger das Bild gestohlen habe, denn dieses stehe auf Grund des Ermittlungsverfahrens nicht fest. Auch hätten sie keinen Beweis dafür angeboten, dass der Kläger das Bild gestohlen habe. Zweifel am Vortrag des Klägers zur Übergabe des Bildes allein genügten nicht. Zur Widerlegung der Eigentumsvermutung müsse vielmehr der volle Beweis des Gegenteils gemäß § 292 ZPO erfolgen. Die Beklagten hätten jedoch keinen hinreichenden Beweis dafür angetreten, dass der Kläger in den Besitz des Bildes durch Diebstahl oder Hehlerei oder eine andere Straftat gekommen sei.

3. Hiergegen wendet sich die Berufung der Beklagten. Das Landgericht habe die Vermutungswirkung des § 1006 BGB und die Voraussetzung für ihre Widerlegung unzutreffend erfasst. Ein Abhandenkommen könne nicht allein mit der Begründung verneint werden, dass die Beklagten keinen hinreichenden Beweis dafür angeboten hätten, dass der Kläger in den Besitz des Bildes durch Diebstahl oder Hehlerei oder eine andere Straftat gelangt sei. Ausreichend sei vielmehr der Nachweis, dass der Eigentümer den Besitz ohne seinen Willen verloren habe. Angesichts der hierfür bereits in erster Instanz vorgetragenen Indiztatsachen habe das Landgericht Beweis erheben müssen. Erst nach Urteilsverkündung sei überdies bekannt geworden, dass auch der Zeuge L3 das Bild noch in den Jahren 2008 oder 2009 und damit nach dem von dem Kläger behaupteten Erwerbszeitpunkt im Atelier des Erblassers gesehen habe. Auch sei der Kläger ein enger Freund des zwischenzeitlich verstorbenen Rechtsanwalts H2. Dieser habe von den Beklagten im Verfahren vor dem LG Köln die Herausgabe zweier Bilder verlangt, die er im Jahr 2010 vom Kläger gekauft habe. Ausweislich eines Gutachtens des Zeugen U vom 5. April 2011 seien diese jedoch unecht.

Die Beklagten beantragen, unter Abänderung der angefochtenen Entscheidung die Klage abzuweisen.

Der Kläger beantragt demgegenüber, die Berufung zurückzuweisen.

Er wiederholt und vertieft sein erstinstanzliches Vorbringen. Das Landgericht habe eine Beweisaufnahme mit Recht abgelehnt, weil die zu beweisenden Behauptungen mangels Konkretisierung einer Beweisaufnahme nicht zugänglich seien. Die von den Beklagten herangezogenen Angaben des Erblassers anlässlich seiner polizeilichen Vernehmung vom 14. Oktober 2009 seien unzutreffend. So sei das streitgegenständliche Bild bereits ausgestellt gewesen und zwar in Amsterdam. Überdies habe die Zeugin Q4 am 2. Januar 2012 gegenüber Rechtsanwalt $C$ angegeben, der Erblasser habe ihr gegenüber im Oktober 2009 eingeräumt, sich nicht sicher zu sein, das Bild nicht doch verkauft zu haben. Die angekündigte Widerlegung der klägerischen Erwerbsgeschichte durch den Zeugen L3 sei demgegenüber ein versuchter Prozessbetrug. Nach Angaben des 
Zeugen werde auf diesen Druck ausgeübt, damit er gegenüber dem Senat Angaben im Sinne der Beklagten mache. Auch der Zeugin Q4 sei nahegelegt worden, sich an ihre Erklärung vom 2. Januar 2012 nicht mehr erinnern zu können. Entgegen dem Vortrag der Berufung sei im Verfahren vor dem LG Köln zu keinem Zeitpunkt behauptet worden, dass der verstorbene $\mathrm{H} 2$ die im dortigen Verfahren streitgegenständlichen Bilder vom Kläger erworben habe.

Die Akten der StA Köln lagen vor und waren Gegenstand der mündlichen Verhandlung. Der Senat hat Beweis erhoben durch Vernehmung der Zeugen L2, T, T2, U, Q4, G, T3, G2, H, T4 und L3.

\section{II.}

Die zulässige, form- und fristgerechte Berufung der Beklagten hat keinen Erfolg. Der Kläger hat aus § 985 BGB gegen die Beklagten einen Anspruch auf Herausgabe des im Tenor näher bezeichneten Bildes des Erblassers. Der Besitz der beklagten Erben ist unstreitig. Er ist mit dem Tod des Erblassers, an welchen die Staatsanwaltschaft das Bild im Jahr 2009 herausgegeben hatte, auf die Erben übergegangen ( 857 BGB). Dass der Kläger Eigentümer des Bildes ist, folgt aus der Vermutung des $§ 1006$ Abs. 2 BGB. Diese Vermutung vermochten die Beklagten nicht zu widerlegen. Ein vom Eigentum abgeleitetes Recht zum Besitz im Sinne von $\S 986$ BGB haben die Beklagten nicht geltend gemacht.

1. Gemäß § 1006 Abs. 2 BGB wird zugunsten eines früheren Besitzers vermutet, dass er während der Dauer seines Besitzes Eigentümer der Sache gewesen sei. Die von dem Besitzerwerb ausgehende Eigentumsvermutung zugunsten des früheren Besitzers wirkt ungeachtet des irreführenden Wortlauts der Bestimmung über die Beendigung des Besitzes hinaus so lange fort, bis sie widerlegt wird (BGH, Urteil v. 25. Januar 1984 - VIII ZR 270/82, NJW 1984, 1456, zitiert juris Rn. 14; v. 19. Dezember 1994 - II ZR 4/94, NJW 1995, 1292, zitiert juris Rn. 16; v. 10. November 2004 - VIII ZR 186/03, BGHZ 161, 90, zitiert juris Rn. 61; jeweils mwN). Da § 1006 BGB - wie insbesondere § 1006 Abs. 1 S. 2 BGB zeigt - auf der vermuteten Gleichzeitigkeit von Besitzerwerb und Eigentumserwerb aufbaut, ist Gegenstand der Vermutungswirkung der Bestimmung nur, dass der frühere oder jetzige Eigenbesitzer beim Besitzerwerb - und aufgrund desselben - Eigentümer geworden ist (BGH, Urteil v. 5. Juli 1967 - VIII ZR 169/65, NJW 1967, 2008, zitiert juris Rn. 14; v. 23. April 1975 - VIII ZR 58/74, NJW 1975, 1269, 1270, zitiert juris Rn. 18; v. 25. Januar 1984, aaO). Die Eigentumsvermutung des § 1006 BGB gilt deshalb nicht, wenn sich aus dem eigenen Vortrag des Besitzers ergibt oder anderweitig festgestellt wird, dass mit dem Erwerb des Besitzes der Eigentumserwerb nicht verknüpft war (BGH, Urteil v. 5. Juli 1967, aaO; v. 25. Januar 1984, aaO Rn. 16). Die Eigentumsvermutung des $\S 1006$ Abs. 2 BGB tritt überdies hinter derjenigen zugunsten des gegenwärtigen Besitzers nach Absatz 1 der genannten Bestimmung zurück (OLG Düsseldorf, Urteil v. 9. März 1994 - 11 U 62/93, NJW-RR 1994, 866, zitiert juris Rn. 3; Staudinger-Gursky, BGB, 2012, § 1006 Rn. 19; Palandt-Bas- senge, BGB, 73. Aufl., § 1006 Rn. 5). Letzteres gilt allerdings nicht, wenn für den späteren Besitzer wegen § 1006 Abs. 1 S. 2 BGB keine Vermutung streitet ( $B G H$, Urteil vom 23. Mai 1956 - IV ZR 310/55, NJW 1956, 1151; Staudinger-Gursky, aaO; Palandt-Bassenge, aaO).

Hiernach streitet die Vermutung des § 1006 Abs. 2 BGB zugunsten des Klägers. Er war bis zum 4. September 2009 unmittelbarer Besitzer des Bildes. Diesen hat er am genannten Tag aufgrund der Beschlagnahme des Bildes durch die Staatsanwaltschaft Köln verloren. Auch soweit die Staatsanwaltschaft den Besitz nach der Beschlagnahme als mittelbaren Besitz aufrechterhielt (vgl. BGH, Urteil v. 14. Januar 1993 - IX ZR 238/91, NJW 1993, 935, zitiert juris Rn. 10; MünchKomm-Joost, BGB, 6. Aufl., § 868 Rn. 41; vgl. auch BGH, Beschluss v. 20. Oktober 1954 - 4 StR 490/54, NJW 1954, 1942, 1943), endete er spätestens mit der aufgrund der Verfügung der Staatsanwaltschaft Köln vom 21. Dezember 2009 erfolgten Herausgabe des Bildes an den Erblasser. Demgegenüber können sich die Beklagten als gegenwärtige Besitzer nicht auf die Vermutungswirkung des $\S 1006$ Abs. 1 BGB berufen. Mit dem Erbfall ging zwar gemäß $\S 857$ BGB der Besitz des Erblassers auf die Beklagten als Erben über, sodass eine etwaige Eigentumsvermutung für sie fortbesteht (vgl. BGH, Urteil v. 14. Januar 1993, aaO Rn. 11; MünchKomm-Joost, aaO § $857 \mathrm{Rn}$. 9). Mit der Herausgabe des Bildes durch die Staatsanwaltschaft und der damit erfolgten Besitzerlangung des Erblassers war ein Eigentumserwerb jedoch nicht verknüpft.

2. Diese Vermutung können die Beklagten nur durch den Beweis des Gegenteils ( 292 ZPO) zu voller - freilich gemäß § 286 ZPO auch aus den Gesamtumständen zu gewinnender - Überzeugung des Gerichts widerlegen (vgl. BGH, Urteil v. 4. Februar 2002 - II ZR 37/00, NJW 2002, 2101, 2102, zitiert juris Rn. 7; v. 20. September 2004 - II ZR 318/02, NJW-RR 2005, 280, zitiert juris Rn. 20). Die Beklagten müssen folglich beweisen, dass der vermutungsbegünstigte Kläger nie Eigentümer geworden ist (BGH, Urteil v. 16. Oktober 2003 - IX ZR 55/02, BGHZ 156, 310, zitiert juris Rn. 23 ff; v. 20. September 2004, aaO). Sie haben daher entweder den Nachweis zu erbringen, dass es zwischen dem Erblasser als ursprünglichem Eigentümer und dem Kläger keine Einigung über einen Eigentumsübergang auf den Kläger gegeben hat, oder dass der Kläger im Zeitpunkt des Erwerbs bösgläubig im Sinne von § 932 Abs. 2 BGB war oder dass das Bild dem Erblasser im Sinne von § 935 Abs. 1 BGB abhanden gekommen war (vgl. BGH, Urteil v. 20. September 2004, aaO). Ein solcher Nachweis ist den Beklagten nicht gelungen.

a) Zwar erscheint die Darstellung des Klägers zur Erwerbsgeschichte des Bildes durchaus fragwürdig. Schon für sich betrachtet bietet der klägerische Vortrag Anlass zu einer kritischen Würdigung. Es liegt nicht nahe, dass der Erblasser den Transport eines seiner Bilder auf der offenen Ladefläche eines Pritschenwagens zugelassen hätte. Dies gilt erst recht angesichts der Angaben des Zeugen $\mathrm{H}$, nach welchen das Bild auf der Ladepritsche durch das Verstellen von Gips-Mörtel-Säcken 
gesichert worden sein soll. Auch sind die angeblichen Bedingungen für einen Verkauf ungewöhnlich. Hiernach sollte der Kläger den Kaufpreis von immerhin 100.000 Euro in bar übergeben und zusagen, das Bild nie in eine Auktion zu geben, es ausschließlich privat zu halten und nur notfalls zu veräußern. Überdies sprechen weitere Umstände gegen den Kläger. Das Bild hatte sich bei der Beschlagnahme entgegen früherer Zeitpunkte auf einem unsachgemäßen Holzrahmen befunden. Wie durch den Zeugen $U$ bestätigt, war es entgegen der ursprünglichen Justierung so auf dem Rahmen befestigt, dass sich Teile der Darstellung der Bildervorderseite auf den Seiten des Rahmens befanden. Es ist nicht zu erwarten, dass ein Künstler sein Bild derart unsachgemäß gerahmt aus der Hand gibt. Gleiches gilt für die mit der üblichen Signatur des Erblassers nicht in Einklang stehende Signatur mit "Q3.“ Der Zeuge U hat diese ausdrücklich nicht dem Erblasser zugeordnet. Der Zeuge T2 hat die Signatur im Einklang zu ähnlichen Ausführungen des Zeugen L2 sogar ausdrücklich als "Witz" bezeichnet. Auch nach seiner Auffassung hätte der Erblasser niemals ernsthaft in dieser Form gezeichnet. Mit Blick auf das von den Zeugen T, L2 und L3 bestätigte Vorbringen der Beklagten, nach welchem nicht nur der langjährig mit dem Erblasser freundschaftlich verbundene $T$, sondern auch andere Sammler immer wieder an einem Ankauf des nämlichen Kunstwerks interessiert waren, der Erblasser dies jedoch stets mit dem Hinweis der Unverkäuflichkeit abgelehnt hatte, erscheint es überdies bemerkenswert, dass ein Verkauf ausgerechnet an den Kläger erfolgt sein soll. Dieser will nur vergleichbar kurz mit dem Erblasser in Kontakt gewesen sein. Es ist in diesem Zusammenhang bezeichnend, dass der Zeuge U den Kaufpreis von 100.000 Euro auch für den Senat nachvollziehbar als gering eingestuft hat. Der Zeuge beschäftigt sich als künstlerischer Leiter des Nachlasses des Erblassers eingehend mit dessen Werken. Bei seiner Einschätzung hat er das zu Lebzeiten des Erblassers bestehende Preisgefüge, die Größe des Bildes und seine künstlerische Bedeutung im Gesamtwerk des Erblassers berücksichtigt. Hiernach wäre im Jahr 2007 ein Preis von 400.000 Euro immer noch angemessen gewesen. Dies entspricht auch der Einschätzung, die der Erblasser anlässlich seiner polizeilichen Vernehmung am 14. Oktober 2009 selbst abgegeben hatte. Überdies hatte der Erblasser nach den Angaben der Zeugen L2 und T2 auch keinen Exklusivvertrag mit einer Galerie geschlossen. Ein solcher Vertrag konnte deshalb für ihn nicht Anlass für die ungewöhnlichen und dem Kläger angeblich vorgegebenen Verkaufsbedingungen sein. Ferner erscheinen nach der Beweisaufnahme auch die Angaben des Klägers zur angeblichen Übergabe des Bildes an den mit dem Transport zum Kläger betrauten Zeugen $\mathrm{H}$ fragwürdig. Zwar hat der vorgenannte Zeuge die klägerische Darstellung bestätigt, nach welcher der Erblasser sich beim Abholen des Bildes in einem Zelt im Hof aufgehalten und dann beim Aufladen geholfen habe. Demgegenüber hat der Zeuge T2 angegeben, er habe nie gesehen, dass der Erblasser auf dem zum Atelier gehörenden Hof herumgelaufen oder in das dort befindliche Zelt gelaufen wäre. Der Erblasser habe sich seit dem Jahr 2007 mit Blick auf seine schwere Krebserkrankung nicht mehr zum Hinterausgang der Halle bewegt, in der er eigentlich gearbeitet hatte. Er sei teilweise nicht mehr aus seinem Bett herausgekommen. Er habe sich auch nicht mehr aufgerafft, um Sachen anzuschauen, die ihn eigentlich interessiert hätten. Auch soll der Erblasser gegenüber dem Zeugen geäußert haben, dass er sich die Aushändigung von wertvollen Gegenständen stets quittieren lasse. Letzteres ist auch nach Angaben des Klägers nicht erfolgt. Unabhängig davon hat auch keiner der Zeugen das Bild in dem Zelt auf dem Hof gesehen, wo es der Zeuge $\mathrm{H}$ abgeholt haben will. Schließlich ist auch die Vernehmung des Zeugen $G$ hinsichtlich des vom Kläger behaupteten Kontakts zum Erblasser unergiebig geblieben.

b) Demgegenüber konnten die Beklagten schon nicht beweisen, dass das nämliche Bild entgegen dem vom Kläger behaupteten Erwerb nebst Übergabe im Jahr 2007 noch in den Jahren 2008 oder 2009 in den Räumlichkeiten des Erblassers vorhanden war. Der Zeuge T, der sich wiederholt für den Ankauf des Bildes interessiert hatte, hat schon keine genauen Angaben dazu machen können, wann er das Bild letztmalig im Atelier gesehen hatte. Es könne 2008, 2007 oder 2009 gewesen sein. Der Zeuge L2 hatte zwar als Speditionskaufmann für den Erblasser gearbeitet und in diesem Zusammenhang wiederholt Zutritt zu dessen Atelier. Auch will er das Bild nach dem Umzug in das vorgenannte Atelier im Jahr 1991 oft gesehen haben. Er hat jedoch nicht angeben können, wann dies genau war. Wirklich wieder wahrgenommen habe er das Bild erst Ende des Jahres 2009. Dies kann zeitlich indes auch nach der Herausgabe des Bildes an den Erblasser liegen. Ebenso unergiebig diesbezüglich sind die Angaben des Zeugen L3. Dieser kannte den Erblasser seit dem Jahr 1979 und verkaufte etwa ab dem Jahr 2001 für diesen Kunstwerke. Der Zeuge hat zwar eine konkrete Erinnerung an das streitgegenständliche Bild, das nach seinen Angaben im Atelier gemeinsam mit anderen großformatigen Werken in der sogenannten Schinkenkammer gestanden habe, und zwar unten rechts. Es sei aufgefallen, weil es sehr groß sei. Zu dem Zeitpunkt, wann er das Bild zum letzten Mal gesehen habe, könne er jedoch keine genauen Angaben machen; er wisse nur, dass das Bild dann wieder da war. Auch die Zeugin Q4, die gemeinsam mit L2 im fraglichen Zeitraum unter anderem damit befasst war, alle Bilder zu fotografieren, hat keine Erinnerung daran, wann sie es gesehen hatte. Demgegenüber haben die Zeuginnen T3 und T4 im Einklang mit den Angaben des Klägers bestätigt, dass sich das Bild ab dem Jahr 2007 in dessen Besitz befunden hatte.

Auch vermag der Senat nicht auszuschließen, dass der Erblasser das nämliche Bild verkauft hatte. Zwar hatte dieser anlässlich seiner polizeilichen Vernehmung am 14. Oktober 2009 ausweislich der Vernehmungsniederschrift angegeben, er habe das Bild wie alle seine Werke, die er nicht oder noch nicht in den Verkauf gegeben habe, nicht signiert; das Bild sei unverkäuflich. In diese Richtung deuten auch die Angaben des Zeugen T. Dieser hatte den Erblasser wiederholt gefragt, ob er ihm das Bild verkaufe, wobei der Erblasser stets geantwortet habe, noch nicht so weit zu sein. Überdies hat auch der Zeuge L2 bestätigt, dass es für das Bild auch einen Interessenten 
aus Skandinavien gab, der sich vergeblich um einen Ankauf bemüht hatte. Nach Angaben des Zeugen U soll der Erblasser üblicherweise nur Werke aus seiner aktuellen Produktion verkauft haben, wozu das nämliche Bild mit Blick auf sein frühes Entstehungsjahr in den 1970er-Jahren nicht gehörte. Diese Einschätzung steht in Einklang mit den Angaben des Zeugen T2, der den Erblasser anwaltlich beraten hatte. Hiernach hatte der Erblasser ihm gegenüber ausdrücklich erklärt, er verkaufe das Bild nicht; es sei unverkäuflich, weil es für ihn persönlich eine große Bedeutung habe. Auch hat der Zeuge, der nach seinen Angaben bei der polizeilichen Vernehmung des Erblassers am 14. Oktober 2009 anwesend war, bestätigt, dass der Erblasser gegenüber den Ermittlungsbeamten Angaben in diesem Sinne gemacht hatte. Überdies habe der Erblasser dem Zeugen gegenüber immer wieder die Frage aufgegriffen, wie das Bild fortgekommen sei. Demgegenüber hat die Zeugin Q4 angegeben, sie habe zwar auf Geheiß des Zeugen T2 für den Erblasser Strafanzeige wegen des Verlustes des Bildes erstattet. Der Erblasser habe ihr gegenüber später jedoch erklärt, er könne nicht sicher sein, ob er das Bild nicht doch verkauft habe. Der Senat verkennt nicht, dass der Zeuge T2 in seiner Vernehmung einen Zusammenhang zwischen dem Inhalt der Aussage der Zeugin Q4 und einer zwischenzeitlichen Auseinandersetzung zwischen den Beklagten und der Zeugin gesehen hat. Die Auseinandersetzung hat die Zeugin in ihrer Vernehmung jedoch offen angegeben. Der "Nachlass" habe behauptet, sie habe ein Bild des Erblassers gestohlen, obwohl dieser es ihr tatsächlich geschenkt habe. Einseitige Aussagetendenzen waren in diesem Zusammenhang zu Lasten der Beklagten nicht erkennbar. Die Angaben der Zeugin werden überdies gestützt durch die Einschätzung des Zeugen L3. Dieser hat einen Verkauf des Bildes durch den Erblasser an den Kläger nicht ausschließen wollen, und zwar vor dem Hintergrund der Persönlichkeit des Erblassers. Dieser sei im positiven Sinne verrückt gewesen. Für ihn sei die Kunst an erster Stelle gekommen, alles andere sei ihm egal gewesen. In seinem sonstigen Leben sei er Anarchist gewesen und habe sich um nichts gekümmert. Geld habe keine Rolle gespielt. Es sei deshalb im Nachhinein sehr schwer einzuschätzen, wie sich der Erblasser verhalten habe. Der Zeuge hatte nach seinen Angaben sogar Situationen erlebt, in welchen der Erblasser wertvolle Bilder für wenig Geld verkauft oder auch verschenkt hatte. Der Erblasser habe Spaß daran gehabt, „zuweilen andere Leute zu verarschen." Er habe "da auch richtig bös sein können." Vor diesem Hintergrund mag der Erblasser zwar sowohl gegenüber den Ermittlungsbeamten der Polizei als auch gegenüber den Zeugen T, L2 und T2 das nämliche Bild als unverkäuflich bezeichnet haben. Mit Blick auf die Angaben der Zeugen Q4 und L3 kann jedoch nicht ausgeschlossen werden, dass er das Bild nicht doch veräußert hatte. Bezeichnenderweise haben sich dessen Angaben vom 14. Oktober 2009 gegenüber den Ermittlungsbeamten auch insoweit als unzuverlässig erwiesen, als das nämliche Bild hiernach noch keine Ausstellung gesehen haben soll. Die Zeugen L2 und T haben demgegenüber übereinstimmend bekundet, das Bild sei im Jahr 1993 in Amsterdam ausgestellt gewesen. c) Im Rahmen der gebotenen Gesamtwürdigung der für und gegen die Behauptung der Beklagten streitenden Umstände (vgl. BGH, Urteil v. 4. Juli 1989 - VI ZR 309/88, VersR 1989, 1063, zitiert Juris Rn. 10; v. 15. Januar 1996 - II ZR 242/94, NJW-RR 1996, 664, zitiert Juris Rn. 9; v. 22. November 2006 - IV ZR 21/05, NJWRR 2007, 312, zitiert Juris Rn. 18) vermag sich der Senat vor diesem Hintergrund keine Überzeugung davon verschaffen, dass das nämliche Bild dem Erblasser gestohlen wurde, es verloren gegangen oder sonst abhanden gekommen war (§935 BGB).

Schon für seine ungewöhnlichen Verkaufsbedingungen kann der Erblasser auch andere Gründe gehabt haben als den Schutz eines sich in der Beweisaufnahme nicht bestätigten Exklusivvertrages mit einer Galerie. Der Zeuge L3 hat nicht ausschließen wollen, dass der Erblasser auch „schwarz" Verkäufe getätigt habe. Dieser sei kein Heiliger gewesen, er habe ebenso Bilder verschenkt oder getauscht. Dies steht im Einklang mit den Angaben der Zeugin Q4, die von dem Erblasser ein Bild geschenkt bekommen haben will. Auch der Zeuge T hat angegeben, man habe beim Erblasser manchmal für kein Geld der Welt ein Bild kaufen können; manchmal sei es aber doch möglich gewesen. Passend zu diesem wechselhaften Verhalten hat der Zeuge L2 davon berichtet, der Erblasser habe, wenn eine Galerie zu stark mit dem Verkauf seiner Werke befasst war, sogar absichtlich andere Galerien beauftragt, um die nämliche Galerie zu ärgern. Auch der Umstand, dass sich das Bild bei der Beschlagnahme entgegen früheren Zeitpunkten auf einem unsachgemäßen Holzrahmen befunden hatte, muss nicht zwingend auf einen Diebstahl oder anderen unfreiwilligen Besitzverlust des Erblassers deuten. Zwar hat der Zeuge $U$ für den Senat nachvollziehbar ausgeführt, dass der Erblasser durchaus ungewöhnliche Rahmen verwendet hatte, der aus BaumarktMaterialien stammende Rahmen am nämlichen Bild hierzu aber schon deshalb nicht gehört hatte, weil das Bild entgegen einer früheren Rahmung unfachmännisch aufgezogen war. Der Zeuge $L 2$ hat demgegenüber schon nicht ausschließen wollen, dass der Erblasser zu irgendeiner Zeit einen Rahmen gefertigt hatte, wie ihn das Bild im Zeitpunkt der Beschlagnahme dann aufgewiesen hatte. Auch mag die mit "Q3" ungewöhnliche Signatur auf das von den Beklagten behauptete Abhandenkommen des Bildes deuten. Der Zeuge L2 hat in Übereinstimmung mit den Angaben des Erblassers im Rahmen seiner polizeilichen Vernehmung vom 14. Oktober 2009 angegeben, dieser habe Bilder grundsätzlich erst signiert, wenn sie verkauft wurden oder bereits verkauft waren. Einen zwingenden Schluss auf den von den Beklagten behaupteten unfreiwilligen Besitzverlust lässt jedoch auch dies nicht zu. Nach Angaben des Zeugen L3 soll der Erblasser Bilder teilweise nicht selbst signiert haben. Bei Ortsabwesenheit soll er teilweise Dritte, bei denen es sich um Personen seines Vertrauens gehandelt habe, mit der Zeichnung beauftragt haben. Überdies soll er in Einzelfällen Bilder sogar ohne Signatur abgegeben haben und dies bewusst, um einen Wiederverkauf zu erschweren. Dies würde auch im Hinblick auf die von den Beklagten in dem nicht nachgelassenen Schriftsatz vom 5. August 2015 angestellten Erwägungen gelten, und zwar auch dann, wenn die Signatur vom gleichen Urheber stammen 
sollte, der die im Gutachten der Sachverständigen I als Fälschung bezeichneten Bilder gefertigt haben soll. Dieser Vortrag - gestützt auf das Gutachten vom 6. November 2014 in einem Verfahren, an dem der Prozessbevollmächtigte der Beklagten beteiligt war - ist erst nach Schluss der mündlichen Verhandlung eingeführt worden, ohne dass die Beklagten hierfür nachvollziehbare Gründe angegeben haben. Er war daher schon nicht zuzulassen. Unabhängig davon zwingt die Annahme, dass der Kläger Kontakt zu einem Fälscher von Q2-Bildern gehabt haben soll, nicht zu dem Schluss, das streitgegenständliche Bild sei entwendet worden. Eine Wiedereröffnung der mündlichen Verhandlung war daher nicht geboten.

Es mag auch einiges dafür sprechen, dass der Erblasser entgegen der Darstellung des Klägers und den Angaben des Zeugen $\mathrm{H}$ aufgrund seines Gesundheitszustandes nicht beim Aufladen des Bildes auf die offene Ladefläche eines Pritschenwagens helfen konnte. Hierfür sprechen die Angaben der Zeugen U und T2 zum Gesundheitszustand des Erblassers im fraglichen Zeitraum, zumal der Erblasser nach Beobachtungen des Zeugen $U$ niemals beim Einpacken oder dem Transport von Bildern geholfen hatte. Sicher erscheint jedoch auch dies nicht. Der Zeuge L2 hat sich jedoch daran erinnert, dass der Erblasser in dem hier interessierenden Jahr 2007 bei einer Ausstellung in Siegen noch selbst größere Bilder bewegt haben soll. Selbst wenn, wie der Zeuge $U$ auf Vorhalt dieser Angaben vermutet hat, dies nur mit dem linken Arm erfolgt sein sollte, kann die Richtigkeit des klägerischen Vortrags hiernach nicht ausgeschlossen werden.

Entscheidend für den Senat ist insbesondere das Bild, das maßgeblich die Zeugen L3, Q4, L2 und T von der Persönlichkeit des Erblassers vermittelt haben. Hiernach handelte es sich um einen Künstler, der für sich eigene Gesetze in Anspruch nahm. Der Zeuge L3 bezeichnete inn insoweit als „Anarchisten." Es liegt hiernach auf der Hand, dass der Erblasser Verkaufsentscheidungen betreffend seine Kunstwerke auch aus dem Moment heraus getroffen und dabei auch nicht zwingend Marktpreise verlangt hatte. Sogar eine bewusst fehlerhafte Signatur durch den Erblasser vermag der Senat insbesondere vor dem Hintergrund der Angaben der Zeugen L2 und L3 nicht auszuschließen. Fernliegend erscheint auch die Annahme, der Erblasser habe ein Werk des hier in Rede stehenden Wertes nur gegen Quittung abgegeben. Insgesamt erscheint das Verhalten des Erblassers nicht eindeutig. (Entscheidung von der Readktion bearbeitet.) 